\title{
Minimally invasive approaches for early gastric cancer in East Asia: current status and future perspective
}

\author{
Takahiro Kinoshita \\ Division of Gastric Surgery, National Cancer Center Hospital East, Kashiwa, Japan \\ Correspondence to: Takahiro Kinoshita, MD, PhD, FACS. Division of Gastric Surgery, National Cancer Center Hospital East, 6-5-1 Kashiwanoha, \\ Kashiwa, 277-8577 Chiba, Japan. Email: takkinos@east.ncc.go.jp.
}

\begin{abstract}
East Asia is well known as a region with higher incidences of gastric cancer compared with the rest of the world. This region has also experienced a rise in the detection of early gastric cancer (EGC) for the past three decades. The success of nationwide screening programs conducted in Japan or South Korea, increasing public awareness of gastric cancer, improved diagnostic ability of gastroenterologists and the aging population of modern societies may all contribute to the increase in EGC detection rates. Along with the increasing diagnosis of EGC, several key advances in the minimally invasive approach to EGC treatment have been made. Endoscopic resection is an ideal procedure for lesions without lymph node involvement, and its indications have expanded based on the results of prospective studies. Laparoscopic surgery with lymph node dissection has been becoming a standard treatment for EGC patients. Additionally, robot-assisted surgery is penetrating the field of gastric cancer surgery as surgeons pursue a more accurate minimally invasive approach with reduced morbidity rates. However, prolonged operation time and high cost remain problems to be solved for robot-assisted surgery. In this context, function-preserving surgery has become ever more important and should be considered as a method to enhance patients' quality of life after a gastrectomy for EGC. Pylorus-preserving gastrectomy or proximal gastrectomy is more frequently employed in East Asia and strategies that employ sentinel node (SN) navigation to personalize function-preserving surgery in clinical practice are emerging as several prospective studies investigate its efficacy.
\end{abstract}

Keywords: Minimally invasive surgery (MIS); early gastric cancer (EGC)

Received: 15 September 2019. Accepted: 16 October 2019.

doi: $10.21037 /$ tgh.2019.10.08

View this article at: http://dx.doi.org/10.21037/tgh.2019.10.08

\section{Introduction}

East Asia is well known as a region with elevated risk for gastric cancer according to the worldwide surveillance (1) presumably due to high incidence of Helicobacter pylori infection and cancer associated dietary habits. Detection of early gastric cancer (EGC) (namely, tumor progression within submucosal layer regardless of lymph node involvement) has steadily increased for the past three decades. The reasons may be the successful implementation nationwide screening programs in Japan or South Korea, increasing public awareness of gastric cancer disease, improved diagnostic ability of gastroenterologists or due to aging societies.
EGC accounts for approximately $50 \%$ of gastric cancer diagnoses in Japan and South Korea, where the adherence to nationwide screening programs have continue to increase since their initial implementation in 1960 and 1999, respectively. Interestingly, as the rate of Helicobacter Pylori infection in the younger generation decreases in Japan, the necessity of nationwide gastric cancer screening is now being questioned and a change in the strategies of early detection screening program is being proposed for the near future (2). Moreover, while the majority of gastric cancers in China are diagnosed in advanced stages of disease, there has been a marked increase in the detection of EGC in the metropolitan districts of China. Given the overall rise in EGC in East Asia, its treatment will continue to be a 
substantial healthcare issue for the entire region.

\section{Endoscopic resection using peroral endoscopy}

Endoscopic resection using peroral endoscopy is the ideal intervention for stomach lesions without lymph node involvement, since gastric function is almost totally preserved without compromising patient survival. According to the 2017 the $4^{\text {th }}$ Japanese Gastric Cancer Association (JGCA) treatment guidelines (3), absolute indications for endoscopic mucosal resection (EMR) or endoscopic submucosal dissection (ESD) are as follows $(4,5)$ : differentiated-type in histology, T1a, ulceration (UL) (-), and $\leq 2 \mathrm{~cm}$ in diameter. Absolute indications for ESD are as follows: (I) differentiated-type, T1a, UL(-), but $>2 \mathrm{~cm}$; (II) differentiated-type, T1a, UL(+), and $\leq 3 \mathrm{~cm}$. Until the recently, lesions of undifferentiated-type, T1a, UL(-), and $\leq 2 \mathrm{~cm}$ were regarded as an expanded indication,. With the positive 5-year long-term outcomes of JCOG1009/1010 trial (A Phase II Clinical Trial of Endoscopic Submucosal Dissection for Early Gastric Cancer of Undifferentiated Type) (6) that confirmed the efficacy of ESD for the expanded criteria; this item will be also included in absolute indications for ESD in the near future.

The JGCA guidelines define the pathological criteria of a curative resection after ESD and any specimen that does not meet the criteria is categorized as a noncurative resection. For a patient who has undergone a noncurative ESD, a gastrectomy with lymph node dissection is recommended based on the potential risk of residual tumor in the stomach and/or lymphatic metastases. While the concern for suboptimal tumor clearance and the actual incidence of lymph node metastasis in patients with additional gastrectomy is reported as between $5.3 \%$ and $9.3 \%(7-11)$. This implies that additional gastrectomy was overtreatment for almost $90 \%$ of the patients. Considering these facts, Hatta W, et al. advocated a Scoring System to Stratify Curability after ESD (eCura system) in 2017 (12). They analyzed a multicenter database of the patients who underwent radical gastrectomy after having failed to meet the curative criteria for ESD. The scoring system assigned three points for lymphatic invasion and 1 point each for tumor size $>30 \mathrm{~mm}$, positive vertical margin, venous invasion, and submucosal invasion $\geq 500 \mu \mathrm{m}$. They classified patients into three lymph node metastasis risk groups: low (0-1 point: $2.5 \%$ risk), intermediate ( $2-4$ points: $6.7 \%)$, and high (5-7 points: $22.7 \%)$. This system is really simple and reproducible, thus currently used extensively in the clinical practice to make decisions to perform additional surgery after ESD. Unquestionably, the indication of ESD will be expanded in the future and the requirement of additional surgery personalized based on the specific parameters of the individual patient's clinical status and disease characteristics.

\section{Laparoscopic surgery}

\section{Laparoscopic distal gastrectomy}

The extent and approach for EGC has been well established by the Japanese guidelines including the acceptance of laparoscopic surgery. If the clinical diagnosis of the tumor exceeds the indication for endoscopic resection, radical gastrectomy with lymph node dissection is recommended by the Japanese guidelines (3). The extent of D1/D1+ lymphadenectomy is recommended for cT1N0, and D2 for cT1 $1 \mathrm{~N}+$ A $2 \mathrm{~cm}$ macroscopic resection margin is suggested in the guidelines, and rapid pathological diagnosis using frozen section should be employed when border of the tumor is uncertain.

EGC has been thought to be an appropriate indication for laparoscopic surgery. Large-scale randomized studies to investigate non-inferiority of laparoscopic distal gastrectomy (LDG) to open distal gastrectomy (ODG) for cStage I gastric cancer have been conducted in Japan (JCOG0912) (13) and South Korea (KLASS01) (14,15), where there are high incidences of early-staged gastric cancer disease provide ample opportunity for robust clinical trials in EGC. In JCOG0912, a Phase III study evaluating the noninferiority of laparoscopic to open distal gastrectomy for cStage Ia or $\mathrm{Ib}$ patients conducted between 2010 and 2013, a total of 921 patients (LDG 462, ODG 459) were enrolled from 33 institutions. Operation time was longer in LDG than in ODG (278 vs. 194 min, $\mathrm{P}<0.001$ ), while blood loss was less (38 vs. $115 \mathrm{~mL}, \mathrm{P}<0.001$ ). No difference was shown in the grade 3 or more surgical complications (3.3\%: LDG, $3.7 \%$ : ODG), and operation-related death in both arms. Relapsefree survival is defined as a primary endpoint, and its noninferiority margin for a hazard ratio was set as 1.54 . The non-inferiority was confirmed in the final analysis, and presented in ASCO 2019 congress. In KLASS01, total of 1,416 patients were randomized to either LDG $(n=705)$ or ODG ( $\mathrm{n}=711)$ between 2006 and 2010. In the per protocol analysis (644 and 612), in the LDG group, the overall morbidity rate was significantly lower (LDG vs. ODG; $13.0 \%$ vs. $19.9 \%, \mathrm{P}<0.001$ ), especially, the wound-related complication rate showed its significant difference $(3.1 \%$ s. 
$7.7 \%, \mathrm{P}<0.001)$. The major intra-abdominal complication ( $7.6 \%$ vs. $10.3 \%, \mathrm{P}=0.095)$ and mortality rates $(0.6 \%$ vs. $0.3 \%, \mathrm{P}=0.687)$ were similar between the two groups. The 5 -year overall survival rates were $94.2 \%$ in LDG and $93.3 \%$ in ODG (log-rank $\mathrm{P}=0.64)$.

Apart from well-designed prospective studies, big-data analyses using a National Clinical Database (NCD) in Japan have provided additional support the safety and efficacy of laparoscopic approach to EGC. Hiki et al. (16) analyzed 5,288 patients who underwent LDG registered in NCD and compared the outcome of these patients with those who underwent ODG. There were no significant differences inhospital deaths or in the number of reoperations, however, the length of hospital stay was significantly shorter in the LDG. The study demonstrated that LDG was more often associated with pancreatic fistulas, and encouraged caution during laparoscopic dissections. Similarly, Yoshida et al. (17) analyzed the data for 70,346 patients registered in the NCD to compare incidences of mortality and morbidities between LDG and ODG in the propensity score matched cohorts, stage I patients (ODG: $n=14,386$, LDG: $n=14,386$ ) and stage II-IV patients (ODG: $n=3,738$, LDG: $n=3,738$ ), respectively. There was no significant difference in mortality rate between the two groups, but operation time was significantly longer in LDG, while blood loss and incidences of wound-related complications were significantly less in the laparoscopic group. Pancreatic fistula was observed more often in LDG ( $1 \%)$ compared to ODG $(0.8 \%)(\mathrm{P}=0.01)$ in stage I patients. These data suggested that surgeons should still carefully perform laparoscopic gastrectomy (LG) in clinical practice particularly when lacking enough experiences.

\section{Laparoscopic total gastrectomy (LTG)}

Total gastrectomy includes more technically demanding operative steps, such as esophagojejunal anastomosis or lymphadenectomy around the splenic artery or the splenic hilum. Therefore, the procedural development or surgeon adoption of LTG was delayed when compared to that of LDG. In Japan, JCOG1401 (a confirmatory phase-III trial to evaluate the safety of LTG) (18) was conducted with the leakage incidence of esophagojejunal anastomosis as the primary endpoint. Two-hundred forty-four eligible patients were registered between 2015 and 2017, and the median operation time was $309 \mathrm{~min}$ and the median estimated blood loss was $30 \mathrm{~mL}$. Grade 2-4 esophagojejunal anastomotic leakage was recognized in $2.5 \%$. The overall incidence of grade 3-4 morbidity was $29 \%$. There was no mortality. The safety of LTG for cStage I was confirmed and regarding the long-term outcomes extrapolated from the results of JCOG0912 trial. In South Korea, KLASS03 trial (a multicenter phase-II trial) (19) was conducted. Between 2012 and 2014, 170 patients were registered. The primary endpoint was set to the incidence of postoperative morbidity and mortality at 30th postoperative days, and the rates were $20.6 \%$ and $0.6 \%$, respectively. Actually $9.4 \%$ had grade III or more complications, and $1.9 \%$ had reoperation. While the results of these clinical trials have robustly demonstrated the feasibility of LTG, several real-world data $(20,21)$ showed high incidences of anastomotic leakage in LTG. Thus, the introduction of LTG in low volume centers and by inexperienced surgeons should be carefully considered and implemented with caution.

\section{Function-preserving surgery}

Function-preserving surgery has become a major strategy to enhance the patients' quality of life after gastrectomy and should be considered for patients undergoing curative surgery for EGC. Laparoscopic pylorus-preserving gastrectomy or proximal gastrectomy is increasingly employed in East Asia. While severe reflux was thought to be a serious problem after proximal gastrectomy limiting its use, several novel anti-reflux methods have been published more recently with promising results in terms of body weight change or control of postoperative reflux even in esophagogastric direct anastomosis (double-flap method or SOFY method) $(22,23)$.

\section{Reduced-port laparoscopic surgery}

Reduced-port laparoscopic surgery for EGC is another group of techniques aimed at providing more cosmetic benefit or less invasiveness. To date, several single-center case reports and single-surgeon's experiences (24-27) have been published, but no well-designed prospective studies have been reported. Kunisaki et al. (27) reported results of a comparison study in a propensity-matched cohort between reduced port gastrectomy versus conventional laparoscopic surgery. Their study showed that operation time was significantly longer, but cosmetic satisfaction was better in reduced port surgery. Five-year survival or relapse-free survival was similar between two groups. Reduced port LG appears to be feasible when performed by well-experienced surgeons. However, concerns over unresolved technical 
limitations during the procedure and the reproducibility of this approach for over-weight patients persist; and surgeons remain skeptical. More accumulation of surgeon experiences and technological breakthrough are indispensable for further progress in reduced port laparoscopic surgery for EGC.

\section{Robot-assisted gastrectomy (RG)}

Another minimally invasive approach to EGC is RG. In robot-assisted surgery, robotic surgical platforms (masterslave manipulator) are used, but the principles of the procedures are same as that of laparoscopic surgery. Robotassisted gastric cancer surgery is widely performed in Japan and South Korea. Many case series evaluating robotassisted gastric cancer surgery have concluded that robotassisted surgery was associated with longer operation time and higher costs. Most studies have failed to provide any clear clinical advantage of RG over LG. One singleinstitution retrospective study performed in Japan suggested that there was an advantage of robotic surgery in reducing postoperative morbidity rates compared with conventional laparoscopic surgery (28). However, but the analysis included over $40 \%$ of advanced cases in the RG cohort. Thus, deducing the clear advantages of robotic surgery over conventional laparoscopic surgery in EGC is difficult from such studies. A multi-institutional, single-arm prospective study ( $\mathrm{n}=330$ patients from 15 institutions) was conducted in Japan to compare morbidity rate of RG with that of conventional LG (29). The eligibility was cStage I/II gastric cancer, and the primary endpoint was set as morbidity (Clavien-Dindo Grade $\geq$ IIIa). The specified hypothesis was that RG could decrease the morbidity rate to less than $50 \%$ of that with LG which had a morbidity rate of $6.4 \%$. In protocol-completed 326 patients, the median operation time and estimated blood loss were $313 \mathrm{~min}$ and $20 \mathrm{~mL}$, respectively. Morbidity rate was significantly reduced in RG $(2.45 \%)$. Based upon this result, the Japanese government decided to cover RG by national health insurance system in April 2018. Meanwhile, to obtain more scientifically sound comparative outcomes between robot-assisted versus laparoscopic gastrectomies, a multicenter randomized controlled trial is close to being launched by JCOG for cStage I/I gastric cancer patients who need distal or total gastrectomy with its primary endpoint set as morbidity rate. There is no doubt the expensive cost of the robotic units is an important problem and a barrier to robot-assisted gastrectomies. The release of more reasonably priced model by the industry is expected in the near future and may help alleviate the issue of cost. Additionally, feasibility of reduced-port RG has been reported from South Korea $(30,31)$. It is unquestionable that robotic system has a great potential to lessen the technical difficulty of laparoscopically complicated procedures, such as reduced-port surgery or hand-suturing anastomosis.

\section{Sentinel node (SN) navigation surgery}

Minimally invasive approaches such as laparoscopic or robotic gastrectomy for EGC have made progress and are increasingly accepted as a standardized strategy by enabling the same extent of gastrectomy or lymphadenectomy as that of open surgery. Basically, incidence of lymph node metastasis is relatively low in EGC, therefore, functionpreserving procedures could be considered to avoid excessive invasiveness to the patients. Therefore, proper application of personalized function-preserving surgery using SN concept has the potential to contribute significantly to patients' quality of life after gastric cancer operations (32). Kitagawa et al. (33) conducted a phase-II trial to evaluate feasibility of SN mapping in Japan. Patient eligibility was cT1/2 gastric cancer with less than $4 \mathrm{~cm}$ in macroscopic diameter. SN mapping was performed by using a dual tracer endoscopic injection method. Gastrectomy with D2 (or modified D2) lymphadenectomy was performed followed by a biopsy of the identified SNs. Of the total 397 patients enrolled in the study, the SN detection rate was $97.5 \%$ and the accuracy of nodal evaluation for metastasis was $99 \%$. Only four false-negative SNs were recorded. Based upon these results, a prospective multicenter trial (UMIN ID: 000014401) involving the development of innovative strategies that combine laparoscopic function-preserving gastrectomy and SN mapping is currently ongoing in Japan in order to assess the survival outcomes and patients' quality of life. This study includes cT1N0 EGCs that exceed indications for ESD and are solitary primary lesions less than $40 \mathrm{~mm}$ in size. Similarly, a Korean study group is also running a prospective multicenter randomized controlled phase III clinical trial (SENORITA trial) to clarify the oncological safety of laparoscopic functionpreserving surgery with $\mathrm{SN}$ basin dissection compared to a conventional LG with lymphadenectomy procedure (34). Additionally, several novel techniques have been reported and include a full-thickness partial gastrectomy using combined laparoscopic and endoscopic approaches with SN basin dissection. Such novel methods have the potential to 
further minimize the extent of invasiveness in the treatment of EGC without lymph node metastasis (35).

\section{Conclusions}

Management of EGC is a substantial healthcare issue in East Asian countries. Preservation of gastric function, lessinvasiveness, and maintaining patients' quality of life are key items in the treatment of EGC. In the future, personalized treatment with precise diagnosis of tumor spreading will be a mainstay in this field.

\section{Acknowledgments}

The author thanks Ms. Tamae Takeuchi for her efforts of proofing and correcting the language of the manuscript.

\section{Footnote}

Conflicts of Interest: The author has no conflicts of interest to declare.

Ethical Statement: The author is accountable for all aspects of the work in ensuring that questions related to the accuracy or integrity of any part of the work are appropriately investigated and resolved.

\section{References}

1. Global Cancer Observatory, World Health Organization. Available online: http://gco.iarc.fr/

2. Mizota Y, Yamamoto S. How long should we continue gastric cancer screening? From an epidemiological point of view. Gastric Cancer 2019;22:456-62.

3. Japanese Gastric Cancer Association. Japanese Gastric Cancer treatment guidelines 2014 (ver.4). Gastric Cancer 2017;20:1-19.

4. Tanabe S, Hirabayashi S, Oda I, et al. Gastric cancer treated by endoscopic submucosal dissection or endoscopic mucosal resection in Japan from 2004 through 2006: JGCA nationwide registry conducted in 2013. Gastric Cancer 2017;20:834-42.

5. Hasuike N, Ono H, Boku N, et al. A non-randomized confirmatory trial of an expanded indication for endoscopic submucosal dissection for intestinal-type gastric cancer (c'T1a): the Japan Clinical Oncology Group study (JCOG0607). Gastric Cancer 2018;21:114-23.

6. K Takizawa, A Takashima, A Kimura, et al. A Phase II
Clinical Trial of Endoscopic Submucosal Dissection for Early Gastric Cancer of Undifferentiated Type: Japan Clinical Oncology Group Study JCOG1009/1010. Jpn J Clin Oncol 2013;43:87-91.

7. Kim ER, Lee H, Min BH, et al. Effect of rescue surgery after non-curative endoscopic resection of early gastric cancer. Br J Surg 2015;102:1394-401.

8. Ryu KW, Choi IJ, Doh YW, et al. Surgical indication for non-curative endoscopic resection in early gastric cancer. Ann Surg Oncol 2007;14:3428-34.

9. Suzuki H, Oda I, Abe S, et al. Clinical outcomes of early gastric cancer patients after noncurative endoscopic submucosal dissection in a large consecutive patient series. Gastric Cancer 2017;20:679-89.

10. Sunagawa H, Kinoshita T, Kaito A, et al. Additional surgery for non-curative resection after endoscopic submucosal dissection for gastric cancer: a retrospective analysis of 200 cases. Surg Today 2017;47:202-9.

11. Yang HJ, Kim SG, Lim JH, et al. Predictors of lymph node metastasis in patients with non-curative endoscopic resection of early gastric cancer. Surg Endosc 2015;29:1145-55.

12. Hatta W, Gotoda T, Oyama T, et al. A Scoring System to Stratify Curability after Endoscopic Submucosal Dissection for Early Gastric Cancer: "eCura system.” Am J Gastroenterol 2017;112:874-81.

13. Katai H, Mizusawa J, Katayama H, et al. Short-term surgical outcomes from a phase III study of laparoscopyassisted versus open distal gastrectomy with nodal dissection for clinical stage IA/IB gastric cancer: Japan Clinical Oncology Group Study JCOG0912. Gastric Cancer 2017;20:699-708.

14. Kim W, Kim HH, Han SU, et al. Korean Laparoendoscopic Gastrointestinal Surgery Study (KLASS) Group. Decreased Morbidity of Laparoscopic Distal Gastrectomy Compared With Open Distal Gastrectomy for Stage I Gastric Cancer: Short-term Outcomes From a Multicenter Randomized Controlled Trial (KLASS-01). Ann Surg 2016;263:28-35.

15. Kim HH, Han SU, Kim MC, et al. Korean Laparoendoscopic Gastrointestinal Surgery Study (KLASS) Group. Effect of Laparoscopic Distal Gastrectomy vs. Open Distal Gastrectomy on Long-term Survival Among Patients With Stage I Gastric Cancer: The KLASS-01 Randomized Clinical Trial. JAMA Oncol 2019;5:506-13.

16. Hiki N, Honda M, Etoh T, et al. Higher incidence of pancreatic fistula in laparoscopic gastrectomy. Real-world 
evidence from a nationwide prospective cohort study. Gastric Cancer 2018;21:162-70.

17. Yoshida K, Honda M, Kumamaru H, et al. Surgical outcomes of laparoscopic distal gastrectomy compared to open distal gastrectomy: A retrospective cohort study based on a nationwide registry database in Japan. Ann Gastroenterol Surg 2017;2:55-64.

18. Katai H, Mizusawa J, Katayama H, et al. Stomach Cancer Study Group of Japan Clinical Oncology Group. Singlearm confirmatory trial of laparoscopy-assisted total or proximal gastrectomy with nodal dissection for clinical stage I gastric cancer: Japan Clinical Oncology Group study JCOG1401. Gastric Cancer 2019;22:999-1008.

19. Hyung WJ, Yang HK, Han SU, et al. A feasibility study of laparoscopic total gastrectomy for clinical stage I gastric cancer: a prospective multi-center phase II clinical trial, KLASS 03. Gastric Cancer 2019;22:214-22.

20. Etoh T, Honda M, Kumamaru H, et al. Morbidity and mortality from a propensity score-matched, prospective cohort study of laparoscopic versus open total gastrectomy for gastric cancer: data from a nationwide web-based database. Surg Endosc 2018;32:2766-73.

21. Kodera Y, Yoshida K, Kumamaru H, et al. Introducing laparoscopic total gastrectomy for gastric cancer in general practice: a retrospective cohort study based on a nationwide registry database in Japan. Gastric Cancer 2019;22:202-13.

22. Hayami M, Hiki N, Nunobe S, et al. Clinical Outcomes and Evaluation of Laparoscopic Proximal Gastrectomy with Double-Flap Technique for Early Gastric Cancer in the Upper Third of the Stomach. Ann Surg Oncol 2017;24:1635-42.

23. Yamashita Y, Yamamoto A, Tamamori Y, et al. Side overlap esophagogastrostomy to prevent reflux after proximal gastrectomy. Gastric Cancer 2017;20:728-735.

24. Kim SM, Ha MH, Seo JE, et al. Comparison of Reduced Port Totally Laparoscopic Distal Gastrectomy (Duet TLDG) and Conventional Laparoscopic-Assisted Distal Gastrectomy. Ann Surg Oncol 2015;22:2567-72.

25. Jeong O, Park YK, Ryu SY. Early experience of duet laparoscopic distal gastrectomy (duet-LDG) using three abdominal ports for gastric carcinoma: surgical technique and comparison with conventional laparoscopic distal gastrectomy. Surg Endosc 2016;30:3559-66.

26. Kunisaki C, Makino H, Yamaguchi N, et al. Surgical advantages of reduced-port laparoscopic gastrectomy in gastric cancer. Surg Endosc 2016;30:5520-8.

27. Kunisaki C, Miyamoto H, Sato S, et al. Surgical Outcomes of Reduced-Port Laparoscopic Gastrectomy Versus Conventional Laparoscopic Gastrectomy for Gastric Cancer: A Propensity-Matched Retrospective Cohort Study. Ann Surg Oncol 2018;25:3604-12.

28. Suda K, Man-I M, Ishida Y, et al. Potential advantages of robotic radical gastrectomy for gastric adenocarcinoma in comparison with conventional laparoscopic approach: a single institutional retrospective comparative cohort study. Surg Endosc 2015;29:673-85.

29. Uyama I, Suda K, Nakauchi M, et al. Clinical advantages of robotic gastrectomy for clinical stage I/II gastric cancer: a multi-institutional prospective single-arm study. Gastric Cancer 2019;22:377-85.

30. Seo WJ, Son T, Roh CK, et al. Reduced-port totally robotic distal subtotal gastrectomy with lymph node dissection for gastric cancer: a modified technique using Single-Site ${ }^{\circledR}$ and two additional ports. Surg Endosc 2018;32:3713-9.

31. Lee JH, Son T, Kim J, et al. Intracorporeal delta-shaped gastroduodenostomy in reduced-port robotic distal subtotal gastrectomy: technical aspects and short-term outcomes. Surg Endosc 2018;32:4344-50.

32. Takeuchi H, Goto O, Yahagi N, Kitagawa Y. Functionpreserving gastrectomy based on the sentinel node concept in early gastric cancer. Gastric Cancer 2017;20:53-9.

33. Kitagawa Y, Takeuchi H, Takagi Y, et al. Sentinel node mapping for gastric cancer: a prospective multicenter trial in Japan. J Clin Oncol 2013;31:3704-10.

34. Park JY, Kim YW, Ryu KW, et al. Assessment of laparoscopic stomach preserving surgery with sentinel basin dissection versus standard gastrectomy with lymphadenectomy in early gastric cancer - a multicenter randomized phase III clinical trial (SENORITA trial) protocol. BMC Cancer 2016;16:340.

35. Hiramatsu Y, Takeuchi H, Goto O, et al. Minimally Invasive Function-Preserving Gastrectomy with Sentinel Node Biopsy for Early Gastric Cancer. Digestion 2019;99:14-20.

doi: $10.21037 /$ tgh. 2019.10 .08

Cite this article as: Kinoshita T. Minimally invasive approaches for early gastric cancer in East Asia: current status and future perspective. Transl Gastroenterol Hepatol 2020;5:20. 